Reprod. Nutr. Dévelop., 1987, 27 (1 B), 209-210.

\title{
Comparaison des quantités de fourrage vert ingérées entre des vaches laitières, des génisses de race laitière, des génisses de race à viande et des moutons
}

J. P. DULPHY, P. D'HOUR (*)

Laboratoire des Aliments,

I.N.R.A. Theix, 63122 Ceyrat, France.

(*) Domaine de La Borie

I.N.R.A. Marcenat, 15190 Condat.

Summary. The voluntary dry matter intake of fresh grass by dairy cows, dairy heifers, beef heifers and sheep was compared for 15 to 17 weeks. The relationships were better between different categories of bovines than between bovines and ovines, but an especially good prediction of the intake of dairy cows was obtained in bovines compared with that measured in sheep.

Les comparaisons de quantités d'herbe ingérées à l'auge par différents types de ruminants sont rares. Demarquilly et Weiss (1971) en ont effectué 64 entre moutons adultes et vaches laitières faibles productrices et 58 entre moutons et bcufs de $450 \mathrm{~kg}$. Nous avons poursuivi ce travail en comparant des moutons, des génisses de un an et des vaches en lactation.

Matériel et méthodes. Des mesures individuelles (regroupées par lot et par semaine) des quantités d'herbe ingérées ont été effectuées sur les domaines d'Orcival et de Marcenat pendant 2 mois (juin-juillet). L'herbe était coupée chaque matin et distribuée à volonté $(5-10 \%$ de refus). II s'agissait de prairie naturelle (1er et $2^{\mathrm{e}}$ cycle) dans les deux domaines, plus des repousses de dactyle à Orcival et de ray-grass anglais à Marcenat. Les vaches laitières (race Holstein) (VL) ont reçu systématiquement $1 \mathrm{~kg}$ de tourteau de soja et colza tanné par jour et une petite quantité de concentré équilibré $(1,5 \mathrm{~kg} / \mathrm{j}$ en moyenne à Orcival et $0,5 \mathrm{~kg} / \mathrm{j}$ à Mar-

TABL. 1. - Caractéristiques moyennes des animaux et des fourrages et quantités ingérées par semaine.

Orcival (8 semaines):

Poids vif moyen $(\mathrm{kg})$

Production de lait à $4 \%$ de MG $(\mathrm{kg})$

Teneur en MS de l'herbe (\%)

Digestibilité de la M.O. (\%)

Ingestibilité g MS/kg $\mathrm{P}^{0,75}$ (moutons)

Quantités de MS ingérées :

- herbe $(\mathrm{kg} / \mathrm{j})$

- herbe + concentré $\left(\mathrm{g} / \mathrm{kg} \mathrm{p}^{0,75}\right)$

Marcenat (9 semaines) :

Poids vif moyen $(\mathrm{kg})$

Production de lait à $4 \%$ de MG $(\mathrm{kg})$

Teneur en MS de I'herbe $(\%)$

Digestibilité de la M.O. (\%)

Ingestibilité g MS/kg $\mathrm{P}^{0,75}$ (moutons)

Quantités de MS ingérées :

- herbe $(\mathrm{kg} / \mathrm{j})$

- herbe + concentré $\left(\mathrm{g} / \mathrm{kg} \mathrm{P}^{0.75}\right)$
Vaches

laitières

Génisses de Génisses de race laitière race à viande

\begin{tabular}{rrrr}
622 & \multicolumn{1}{c}{380} & \multicolumn{2}{c}{383} \\
23 & & - & - \\
$18,4 \pm 3,3$ & $18,8 \pm 3,7$ & $18,5 \pm 3,9$ \\
$70,8 \pm 3,9$ & $70,0 \pm 5,0$ & $70,8 \pm 5,0$ \\
$74,4 \pm 7,5$ & $70,4 \pm 8,4$ & $68,9 \pm 10,0$ \\
& & & \\
$16,4 \pm 1,7$ & $7,7 \pm 0,8$ & $6,6 \pm 0,4$ \\
$142 \pm 10,0$ & $89,0 \pm 8,2$ & $77,0 \pm 5,6$
\end{tabular}

\begin{tabular}{rrrrr}
579 & \multicolumn{1}{c}{395} & \multicolumn{2}{c}{366} \\
19 & - & - \\
$17,4 \pm 1,8$ & $17,4 \pm 1,8$ & $17,4 \pm 1,8$ \\
$67,9 \pm 3,1$ & $67,9 \pm 3,1$ & $67,9 \pm$ & 3,1 \\
$69,7 \pm$ & 5,1 & $69,7 \pm 5,1$ & $69,7 \pm$ & 5,1 \\
& & & & \\
$15,3 \pm 1,3$ & $7,6 \pm 1,0$ & $6,2 \pm 0,9$ \\
$135 \pm$ & 7,0 & $85,6 \pm 9,6$ & $74,5 \pm 10,2$
\end{tabular}


cenat) pour couvrir leurs besoins de production laitière. Les génisses de race laitière $(\mathrm{GL})$ étaient des croisées Frisonnes $\times$ Holstein à Orcival, des Montbéliardes et des croisées Frisonnes $\times$ Holstein à Marcenat. Les génisses de race à viande (GV) étaient des Charolaises à Orcival et des Limousines à Marcenat. Nous avons pu regrouper les résultats d'ingestion des génisses car ils étaient les mêmes par rapport au poids métabolique des animaux, pour des caractéristiques identiques de l'herbe. Enfin les moutons (M) étaient des béliers castrés de race Texel de 60-70 kg, placés en cage à métabolisme pour mesurer également la digestibilité de la matière organique des fourrages étudiés. II y avait 6 animaux par lot contre 10 pour chaque type de bovins.

\section{Résultats et discussion.}

Les quantités de MS d'herbe (+ concentré équilibré pour les vaches) ingérées par les bovins $\left(\mathrm{Ol}\right.$ en $\mathrm{g}$ de $\mathrm{MS} / \mathrm{kg} \mathrm{P}^{0,75}$ ) ont été exprimées en fonction des quantités ingérées (QI) par les moutons et des teneurs en matière sèche de l'herbe (MS en \%) ainsi que des productions de lait à $4 \%$ de MG (PL4 en $\mathrm{kg} / \mathrm{j}$ ) :

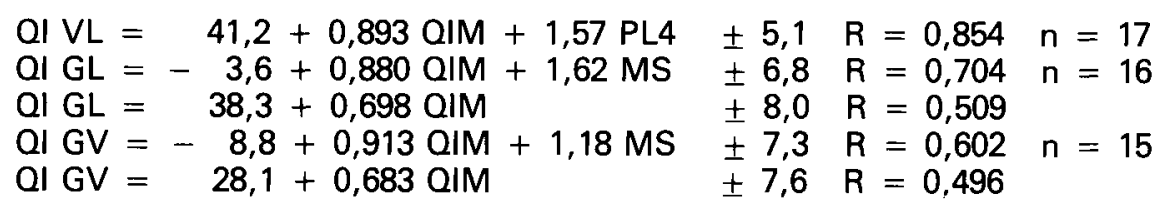

La teneur en MS a, habituellement, un effet notable (Vérité et Journet, 1970). Son addition n'apporte pas d'amélioration significative pour les vaches. Elle en apporte une pour les génisses de race laitière et, sans être significative, améliore notablement le coefficient de corrélation pour les génisses de race à viande. En outre les quantités ingérées par les vaches sont liées à celles ingérées par les génisses :

Ql $V L=46,3+0,723 \mathrm{Q} \mid \mathrm{GL}+1,36 \mathrm{PL} 4 \pm 4,3 \quad \mathrm{R}=0,900 \quad \mathrm{n}=16$

$\mathrm{Q} \mid \mathrm{VL}=36,3+0,896 \mathrm{QIGV}+1,20 \mathrm{PL} 4 \pm 4,9 \quad \mathrm{R}=0,865 \quad \mathrm{n}=15$

Dans ces relations l'introduction de la production laitière améliore significativement le coefficient de corrélation, mais ce n'est pas le cas pour la teneur en MS dont l'incidence est négligeable. Enfin les quantités ingérées par les génisses laitières et à viande sont liées étroitement entre elles de la façon suivante :

$\mathrm{Q}$ I $\mathrm{GV}=-3,2+0,910 \mathrm{Q} \mid \mathrm{GL} \pm 3,0 \mathrm{R}=0,939 \mathrm{n}=15$.

On constate donc de bonnes liaisons entre les quantités ingérées par les vaches d'une part, les génisses et les moutons d'autre part, mais à condition d'additionner le concentré au fourrage vert. Si on n'ajoute pas le concentré équilibré à l'herbe, les relations entre QIVL d'une part, QIM, QIGL et QIGV d'autre part (PL4 étant dans toutes les relations) ont des écarts-types bien plus élevés : $\pm 9,5 ; \pm 6,8 ; \pm 8,4$. Les liaisons sont meilleures si on tient compte de la production laitière.

Les liaisons entre les génisses et les moutons sont plus lâches. Par contre les liaisons entre les quantités ingérées pour les différents bovins sont excellentes, les mécanismes régulateurs de l'ingestion à court terme étant évidemment plus proches à l'intérieur d'une même espèce.

Soulignons enfin la consommation plus élevée des génisses laitières en accord avec les résultats d'Agabriel et al. (1987).

Agabriel J., D'Hour P., Petit M., 1987. Reprod. Nutr. Dévelop., 27, 211-212.

Demarquilly C., Weiss Ph. 1971. Ann. Zootech., 20, 119-134.

Dulphy J. P., Michalet-Doreau B., 1983. Ann. Zootech., 32, 465-474.

Vérité R., Journet M., 1970. Ann. Zootech., 19, 255-268. 